\title{
Cirurgia de revascularização do miocárdio através de minitoracotomia ântero-lateral esquerda
}

\author{
J. Glauco LOBO FILHO *, Francisco M. de OLIVEIRA*, Ciro CIARLINE* ${ }^{*}$ J. Acácio FEITOSA*, \\ Ana Virginia ROLIM*${ }^{\star}$, J. Erirtônio FACANHA*, Roberto A. C. de M. LOBO*, \\ M. Chirstian B. R. DANTAS*, Ricardo de Carvalho LIMA**, Mozart A. S. de ESCOBAR ** \\ José Teles de MENDONÇA ${ }^{* * *}$, José WANDERLEY NETO ${ }^{* * * * *}$
}

RBCCV 44205-302

\begin{abstract}
Lobo Filho J G, Oliveira F M, Ciarline C, Feitosa J A, Rolim A V, Façanha J E, Lobo R A C M, Dantas M C B R, Lima A C, Escobar M A S, Mendonça J T. Wanderley Neto J - Cirurgia de revascularizaçăo do miocárdio através de minitoracotomia ântero-lateral esquerda. Rev Bras Cir Cardiovasc 1996; 11 (3): $143-7$

RESUMO: No periodo de outubro de 1995 a fevereiro de 1996,16 pacientes selecionados foram submetidos a cirurgia de revascularizaçăo do miocárdio através de minitoracotomia ântero-lateral esquerda. Em todos os casos a artéria torácica interna esquerda foi dissecada, para posterior anastomose com o ramo interventricular anterior (RIA) sem a utilizaçăo de circulação extracorpórea. A idade variou de 43 a 77 , com média de 60 anos. Sessenta e dois por cento dos pacientes eram do sexo masculino. Näo houve complicaçōes tais como: hemorragias, acidente vascular cerebral, insuficiência renal aguda, mediastinite ou infarto agudo do miocárdio. Não houve mortalidade no grupo em questăo. Em $4(25 \%)$ pacientes foi realizado estudo hemodinámico, que demostrou uma normalidade da anastomose da artéria torácica interna para o ramo interventricular anterior. Devido aos excelentes resultados iniciais, acreditamos que este procedimento possa ser empregado com maior frequêencia e com a familiarização dos grupos cirúrgicos, e que as artérias diagonais e marginais da circunflexa possam ser beneficiadas com este tipo de procedimento.
\end{abstract}

DESCRITORES: Revascularizaçăo do miocárdio, métodos. Toracotomia, métodos. Artérias torácicas, cirurgia.

\section{INTRODUÇĀO}

A revascularização do miocárdio (RM) por toracotomia antero-lateral esquerda năo é uma técnica nova. VINEBERG (1), em 1946, utilizando esta técnica, introduziu a implantaçăo da artéria torácica interna esquerda (ATIE) no músculo cardíaco sem utilizar circulaçăo extracorpórea (CEC). KOLESSOV (2), em 1967, pela mesma abordagem cirúrgica, desen- volveu uma técnica para a anastomose da ATIE com ramo interventricular anterior (RIA), também sem utilizar CEC. Com a maior compreensão dos danos causados pela CEC (3.7), alguns centros desenvolveram técnicas para cirurgia de RM sem CEC ${ }^{(8,9)}$, principalmente naqueles pacientes portadores de outras doenças orgånicas, tais como insuficiência renal crônica, doenças pulmonares, acidente vascular cerebral (AVC) prévio, idade supe-

Trabalho realizado no Instituto do Coraçåo e Pulmão - ICORP - em cooperação com o Hospital Prontocárdio, Hospital António Prudente - Fortaleza, Ceará - UNITORAX, Pernambuco - Unidade Cardiotorácica de Sergipe e IDC, Alagoas, Brasil.

Apresentado ao $23^{\circ}$ Congresso Nacional de Cirurgia Cardiaca. Recife, PE, 20 a 23 de marco, 1996.

do ICORP

". dO UNITORAX

$\cdots$ da Unidade Cardiotorácica de Sergipe

*** do IDC

Endereço para correspondência: J. Glauco Lobo Fitho. Rua Dr. José Lourenço, 625. Fortaleza, Ceará, Brasil. CEP; 60115-280. 
Lobo Filho J G, Oliveira F M, Ciarline C, Feitosa J A, Rolim A V, Façanha J E, Lobo R A C M, Dantas M C B R, Lima R C, Escobar M AS, Mendonça JT. Wanderley Neto J-Cirurgia de revascularizaçăo do miocárdio através de minitoracotomia ântero-lateral esquerda. Rev Bras Cir Cardiovasc 1996: 11 (3): 143-7.

rior a 75 anos, ou seja, pacientes considerados de "alto risco" $(10,11)$. A familiarizaçăo dos grupos cirúrgicos com esta técnica possibilitou que novas iniciativas surgissem no sentido de promover a RM de maneira pouco invasiva, através de uma minitoracotomia esquerda com dissecção da ATIE para revascularizar o RIA. O objetivo deste estudo é descrever a técnica e analisar os resultados imediatos do referido procedimento.

\section{CASUISTICA E MÉTODOS}

Entre outubro de 1995 a fevereiro de 1996, 16 pacientes foram submetidos a cirurgia de revascularizaçăo direta do miocárdio, sem CEC, através de minitoracotomia ântero-lateral esquerda pelo $4^{2}$ espaço intercostal. A idade variou de 43 a 77 anos com média de 60 anos. Sessenta e dois por cento dos pacientes eram do sexo masculino. Os detalhes da técnica cirúrgica envolveram aspectos que serảo abordados. Realizadas anestesia e monitorização eletrocardiográfica $\Theta$ da pressāo arterial, o paciente é colocado em decúbito lateral direito a $60^{\circ}$. A toracotomia ântero-lateral esquerda é realizada através de uma incisăo em forma semicircular, começando ao nivel da borda esternal esquerda e prolongando-se $12 \mathrm{~cm}$ a $15 \mathrm{~cm}$ acompanhando a silhueta mamária inferior. Após separação dos arcos costais, utilizando-se um afastador de Finochietto, a ATIE é dissecada desde a sua origem até $07^{\circ}$ espaço intercostal, efetuando-se a ligadura de todos os seus ramos com clipe metálico. Neste momento o paciente é anticoagulado, aplicando-se heparina sódica por via intravenosa na dose de $2 \mathrm{mg} / \mathrm{kg}$ de peso, mantendo-se esta heparinizaçäo com a administração de metade da dose inicial a cada hora. Efetua-se a secção da ATIE em sua porção mais distal com abertura da sua luz para posterior anastomose com o RIA. Procede-se, entāo, à abertura do pericárdio, com incisāo longitudinal anterior ao nervo frênico entre $8 \mathrm{~cm}$ a $10 \mathrm{~cm}$, o qual é reparado e fixadas as suas bordas nos campos cirúrgicos. Feita a escolha do local ideal no RIA para se realizar a anastomose, o mesmo é dissecado. O garroteamento a montante e a justante do local da anastomose é realizado passando-se um fio Prolene 4-0 em oito envolvendo a coronária, protegendo-a com um pequeno segmento $(1 \mathrm{~cm})$ de sonda de Folley $n^{2} 10$. Realiza-se a abertura do RIA com uma incisảo longitudinal de aproximadamente $6 \mathrm{~mm}$. Efetua-se, a seguir, a anastomose da porção distal da ATIE com do RIA, em sutura continua com o fio Prolene 7-0. Após o término da anastomose, săo feitos testes para se avaliar as condições da anastomose $\theta$, em seguida, fixar 0 tecido periférico da ATIE no epicárdio. Algumas vezes, utilizou-se verapamil ou propranolol por via intravenosa, para diminuir a fre- qüência cardíaca no momento da anastomose. A heparina é neutralizada com injeçăo por via intravenosa de cloridrato de protamina na dose de $1,0 \mathrm{mg}$ para cada mg de heparina utilizado. Finaliza-se, entäo, com fechamento parcial do pericárdio, revisão rigorosa da hemostasia, drenagem e fechamento do tórax.

\section{RESULTADOS}

A média de permanência hospitalar foi de 4,5 dias. Não houve complicaçōes tais como: hemorragia, AVC, insuficiência renal aguda, mediastinite e infarto agudo do miocárdio. Um paciente apresentou infecçăo da ferida cirúrgica no $10^{2}$ dia de pósoperatório, que evoluiu favoravelmente apenas com drenagem. Quatro (25\%) pacientes, os 3 primeiros e o último foram submetidos a estudo angiográfico no pós-operatório imediato, demostrando perfeitas

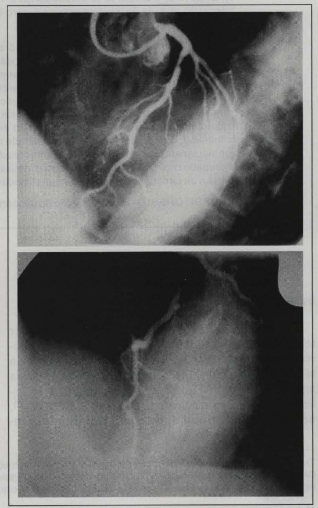

Fig. 1. Angiografia no pré e pós-operatório de revascularização do miocárdio através de minitoracotomia ântero-lateral esquerda, mostrando ponte da ATIE para o RIA o qual exibe uma obstrução de $90 \%$ próximo à sua origem. 
Lobo Filho J G, Oliveira F M, Ciarline C, Feitosa J A, Rolim A V, Façanha J E, Lobo R A C M, Dantas M C B R, Lima R C, Escobar MAS, Mendonça J T. Wanderley Neto J-Cirurgia de revascularização do miocárdio através de minitoracotomia ântero-lateral esquerda. Rev Bras Cir Cardiovasc 1996; 11 (3): 143-7.

condições da anastomose (Figura 1). No primeiro caso operado houve lesāo da ATIE ao nivel do $4^{\circ}$ espaço intercostal, com a artéria curta para alcancar sua finalidade. Por este motivo, sem grandes dificuldades técnicas, foi interposto um segmento de veia safena de aproximadamente $6 \mathrm{~cm}$ entre a extremidade da ATIE e o RIA. Em todos os outros casos a ATIE foi anastomosada diretamente com o RIA. Năo houve mortalidade no grupo estudado.

\section{COMENTÁRIOS}

É consenso, hoje, a preocupação com táticas e técnicas operatórias para tornar os procedimentos menos agressivos. Não existem dúvidas de que a cirurgia de RM, principalmente com 0 uso da ATIE para o RIA é um método eficaz e seguro no tratamento da insuficiência coronária ${ }^{(12)}$. A experiência de vários grupos com a cirurgia de RM sem CEC tem mostrado de uma maneira geral a superioridade deste método em relação à "convencional" com CEC ${ }^{10}$. A reintroduçāo da cirurgia de RM através de uma toracotomia ântero-lateral esquerda merece a análise e algumas considerações importantes. É perfeitamente compreensível que, da época de Kolessov até os dias atuais, houve um profundo desenvolvimento tanto das técnicas operatórias, como do instrumental cirúrgico. Do ponto de vista anatômico, acreditamos ser a cirurgia de preferência para os grupos que têm experiência com a cirurgia de RM sem CEC, facilmente realizável, talvez com um pouco mais de dificuldade para a dissecção da ATIE. Esteticamente, é indiscutível o valor desta técnica, principalmente nas mulheres, onde a incisāo torna-se praticamente imperceptivel (Figura 2). Os excelentes resultados iniciais nos levam a crer que esta técnica possa ampliar as indicações cirúrgicas nos casos de lesāo única do RIA com melho-

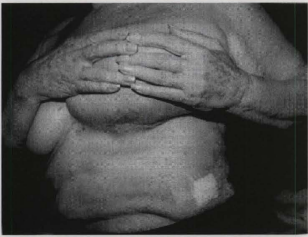

Fig. 2. Aspecto estético da cicatríz no sétimo dia de pós-operatório. res resultados e menores custos que a angioplastia, principalmente no nosso meio. Reflexões deverāo ser feitas ainda a favor da indicaçāo deste procedimento em pacientes de "alto risco" portadores de lesöes multiarteriais coronarianas onde, uma vez realizada a revascularizaçăo do RIA, a angioplastia das outras artérias torna-se mais exequível. Acreditamos também que, com a familiarizaçăo do grupo cirúrgico, as artérias diagonais e posteriores do coraçăo possam ser beneficiadas realizando-se anastomose em $Y$ com a ATIE (13), utilizando-se segmentos arteriais e/ou venosos (Figuras 3 e 4). Ressaltamos a seleçăo criteriosa dos pacientes a serem submetidos a esta técnica e que, preferencialmente, os grupos habituados com a cirurgia de RM sem CEC devem utilizá-la.

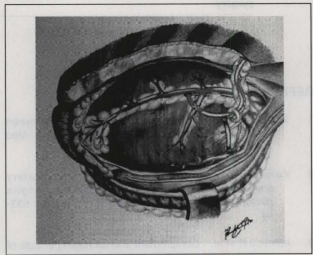

Fig. 3 - Ponte de ATIE seqû̉ncial RIA $\rightarrow$ diagonal, associada com ponte de salena para arteria marginal da circunflexa, fazendo-se anastomose em $Y$ com a ATIE

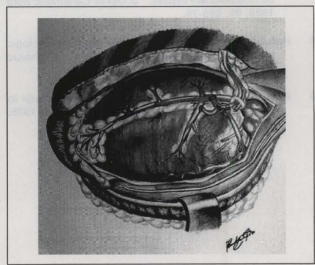

Fig. 4 - Ponte da ATIE para o RIA associada com pontes de segmentos arterias para artéria diagonal e artéria marginal da circunflexa, fazendo anstomose em Y com a ATIE. 
Lobo Filho J G, Oliveira F M, Ciarline C, Feitosa J A, Rolim A V, Façanha J E, Lobo R A C M, Dantas M C B R, Lima R C, Escobar M A S, Mendonça J T. Wanderley Neto J-Cirurgia de revascularizaçäo do miocárdio através de minitoracotomia ântero-lateral esquerda. Rev Bras Cir Cardiovasc 1996; 11 (3): 143-7.

RBCCV 44205-302

Lobo Filho J G, Oliveira F M, Ciarline C, Feitosa J A, Rolim A V, Façanha J E, Lobo R A C M, Dantas M C B R, Lima R C, Escobar M A S, Mendonça J T, Wanderley Neto J-Myocardial revascularization surgery through left anterolateral minithoracotomy. Rev Bras Cir Cardiovasc 1996; 11 (3): 143-7.

ABSTRACT: Between October 1995 and February 1996, sixteen patients were selected to undergo to surgical myocardial revascularization through left anterolateral minithoracotomy. The left internal thoracic artery was dissected in all patients, for consecutive anastomosis with interventricular anterior artery, without using extracorporeal circulation. Patients age ranged from 43 to 77 , average 60 years. Sixty-two percent of them were men. There were no complications such as: acute myocardial infarction, mediastinitis, acute renal failure, hemorrhagy or stroke. There were no deaths. Four $(25 \%)$ patients were submitted to cardiac catheterization that showed patency of grafts and grafted native arteries. Due to excellent initial results, the authors believe that this technique can be employed with greater frequency and that its use can be extended to the treatment of diagonal branches of the interventricular anterior artery and marginal branches of the circunflex, as soon as the surgical teams become more familiarized with it.

DESCRIPTORS: Myocardial revascularization, methods. Thoracotomy, methods. Thoracic arteries, surgery.

\section{REFERÊNCIAS BIBLIOGRÁFICAS}

1 Vineberg A M - Development of anatomosis between coronary vessels transplanted mammary artery. Med Assoc J 1954; 71: 594-602.

2 Kolessov V I - Mammary artery-coronary artery anastomosis as a method of treatment for angina pectoris. J Thorac Cardiovasc Surg 1967; 54: 533 44.

3 Kirklin J K et al. - Complement and damaging effects of cardiopulmonary bypass. JThorac Cardiovasc Surg 1983; 86: 845-57.

4 Blauth C I et al. - Cerebral microembolism during cardiopulmonary bypass. $J$ Thorac Cardiovasc Surg 1988; 95: 668-76.

5 Hammeke T A \& Hastings J E - Neuropsychologic alterations after cardiac operations. J Thorac Cardiovasc Surg 1988; 96: 396-31.

6 Ankeney $\mathrm{J} \mathrm{L}$ - To use or not to use the oxygenator in coronary bypass operations. Ann Thorac Surg 1975 . 19: $188-9$.
7 Butler $\mathrm{J}$ et al. - Inflamatory response to cardiopulmonary bypass. Ann Thorac Surg 1993; 55: 553-9.

8 Buffolo E, Andrade J C S, Succi J B, et al - Revascularizaçāo do miocárdio sem circulaçăo extracorpórea: descrição da técnica e resultados iniciais. Arq Bras Cardiol 1983; 41 : $309 \cdot 16$

9 Buffolo E, Andrade J C, Succi J F et al - Direct myocardial revascularization without cardiopulmonary bypass. Thorac Cardiovasc Surgeon 1985; 33: 26-9.

10 Benetti F J, Naselli G, Wood M, Geffner L - Direct myocardial revascularization without extracorporeal circulation. Prensa Med Argentina 1991; 100: 312-16.

11 Lima R et al. - Revascularizaçăo do miocárdio sem circulação extracorpórea. An Fac Med CCS UFPE (Recife) 1993; 38: 33.7.

12 Cameron A, Davis K B, Green G, Schaff H V - Coronary bypass surgery with internal-thoracic-artery grafts: effects on survival over a 15-year period. N Engl $J$ Med 1996; 334: 216-9.

13 Loop F D - Internal-thoracic-artery grafts. $N$ Engl J Med 1996; 334: 263.5 


\section{Discussão}

\section{DR. SÉRGIO ALMEIDA DE OLIVEIRA}

\section{São Paulo, SP}

Parabéns ao Dr. Glauco e colaboradores, pelo ótimo trabalho. Eu gostaria de fazer duas perguntas e um comentário. Por que houve necessidade de homoenxerto em 3 dos seus pacientes? Por que não realizou estudo cineangiográfico pós-operatório em todos os pacientes? Creio que, no inicio de uma experiência, precisamos de ter informaçōes apuradas sobre as condiçōes funcionais e técnicas do enxerto e da anastomose. Desde novembro de 95, realizamos, em nosso Serviço no Hospital da Beneficenncia Portuguesa e no Hospital Albert Einstein, uma série de 15 casos de revascularizaçāo com minitoracotomia e sem circulaçâo extracorpórea. Em 14 lesões eram isoladas e proximais da artéria interventricular anterior (AIA) e, em 1 caso, a lesão proximal da artéria coronária direita $(C D)$. Este último fora submetido a prévia angioplastia com implante de "stent", sem sucesso. Nāo houve complicação cardiovascular, ocorrendo apenas, em 1 paciente, um pneumotórax espotâneo no lado oposto ao da toracotomia, curado após drenagem pleural. Treze pacientes [12 com anastomose de artéria torácica interna esquerda (ATIE) para a artéria interventricular anterior e $1 \mathrm{com}$ anastomose da ATID para a CD] foram reoperados, com angiografia pós-operatória. Todas as ATI estavam pérvias e com bom fluxo para as artérias coronárias. Em 2 pacientes o estudo do fluxo com Eco-Doppler ao nivel dos $2^{2}$ e $3^{2}$ espaços intercostais direito e esquerdo mostrou sinais patognomónicos e funcionantes da anastomose; não houve redução do fluxo sistólico e aumento do fluxo diastólico, comparativamente às artérias torácicas internas. Portanto, estamos até agora contentes com os resultados desta técnica iniciada por Benetti e que nos foi divulgada por Calafiori, no ano passado, durante o Congresso da SBC. Para casos selecionados, esta técnica é uma boa alternativa cirúrgica. Resta-nos saber se, a longo prazo, esta técnica terá os mesmos bons resultados que obtivemos com a anastomose da ATIE com a artéria IVA. No estudo Mass (J. Am. Coll. Cardiol 26 (7): 1600, 1995, que publicamos recentemente, os 70 pacientes do grupo cirúrgico, mais os 15 que passaram dos outros grupos para posterior cirurgia, vimos que, na cineco. ronariografia realizada ao final de 2 anos de pósoperatório, em todos os pacientes havia apenas uma artéria torácica interna ocluída, dando $98,9 \%$ de perviabilidade. Muito Obrigado.

\section{DR. GLAUCO \\ (Encerrando)}

Em relaçāo à hemotransfusão, é rotina, no nosso Serviço, a administração de concentrado de hemácias nos pacientes com hemoglobina menor ou igual a $9 \mathrm{mg} / \mathrm{dl}$. Tendo em vista o reestudo hemodinåmico, realmente nāo houve uma grande preocupação nesse sentido, uma vez que, ao iniciarmos este procedimento, já haviamos realizado 174 enxertos de ATIE para DA sem CEC, através de esternotomia mediana. Hoje, contudo, a fim de mostrarmos de maneira mais concreta a eficácia deste método, passamos a estudar o máximo possível todos os pacientes. Tanto isso é fato que, dos 43 pacientes operados até a presente data (12 meses), dos 27 últimos, 20 ( $75 \%$ ) foram reestudados, com somente 1 caso apresentando obstrução ao nível da anastomose. Por fim, parabenizamos o Dr. Sérgio Almeida pelos seus comentários e resultados iniciais, e acreditamos que a bem sucedida experiência nacional iniciada por Buffolo e colaboradores, em setembro de 1995, e por nossa equipe em outubro do mesmo ano, nos encoraja a pensar num futuro muito promissor para esta técnica cirúrgica, como também para a operação de RM sem CEC de uma maneira geral. 